\title{
Postalische Kommunikationen. Medizin in der Korrespondenz von Johann Jakob Scheuchzer (1672-1733)
}

Michael Kempe

\section{Summary}

This article examines different aspects of the history of medicine in the correspondence of the Swiss physician and scientist Johann Jakob Scheuchzer (1672-1733) from Zurich. The focus is on three different levels: persons, contents and functions. On all three levels we can observe that "medicine" takes in some way part of the res publica litteraria. The international correspondence network of Scheuchzer can be seen as a crucial "multiplicator" of communication within the scientific community.

Keywords: medical correspondence; correspondence networks; Johann Jakob Scheuchzer (1672-1733); Republic of Letters

\section{Zusammenfassung}

Der Artikel untersucht medizingeschichtliche Aspekte in der Korrespondenz des Zürcher Mediziners und Naturforschers Johann Jakob Scheuchzer (1672-1733) auf drei Ebenen: Personen, Inhalte und Funktionen. Es zeigt sich, dass auf allen drei Ebenen das «Medizinische» jeweils als ein Teilbereich gelehrter Korrespondenz innerhalb der res publica litteraria anzusehen ist. Netzwerktheoretisch betrachtet, entspricht Scheuchzers internationales Korrespondenzgeflecht einem Kommunikationsmultiplikator der scientific community.

Dr. Michael Kempe, Max-Planck-Institut für europäische Rechtsgeschichte, Hausener Weg 120, D-60489 Frankfurt am Main (kempe@mpier.uni-frankfurt.de). 
Ich habe mir vorgenommen durch disse geringfüge und ontüchtig teütsche Zeilen Ihre vilfältigen und ernsthaften geschäfte zu unterbrechen, auch nit durch weitläufige und schmuckreiche lobreden, das helle haus Ihnen erleuchten, modistie, und merites einicher weise unter dem schein eines mehreren glanzes zu verdunklen, sondern nur aus aufrichtigem gemüth sowol Ihre geneigte affection auszubitten, als meiner möglichsten erkantnuss und dienstwilligkeit versicherung zu geben. ${ }^{1}$

Mit diesen - heute umständlich wirkenden - Zeilen beginnt ein Briefwechsel zwischen zwei Schweizer Medizinern, ein reger und inniger Briefwechsel, der fast ein Vierteljahrhundert dauerte und insgesamt über 250 ausgetauschte Schreiben umfasst. Gemeint ist der Briefwechsel zwischen Johann Jakob Scheuchzer (1672-1733) und Theodor Zwinger III (1658-1724). Die weitverzweigten Briefverbindungen des Mediziners und Universalgelehrten Scheuchzer stehen im Zentrum folgender Ausführungen, analysiert werden sollen sie unter dem Stichwort «Medizin im gelehrten europäischen Brief». Dabei werden verschiedene Perspektiven eingenommen, um sich diesem Themenfeld zu nähern. Die Darlegungen verstehen sich als eine erste Bestandsaufnahme, nicht als erschöpfende Untersuchung. Sie sollen auf den Gegenstandsbereich aufmerksam machen und zu weiteren Forschungen über den Scheuchzer-Briefwechsel anregen.

Obgleich einige Briefe und Briefwechsel Scheuchzers gedruckt und kommentiert vorliegen, ist dessen gelehrte Korrespondenz als Ganzes bislang unerforscht; das gilt im besonderen auch für den medizinischen Briefwechsel. Erste Vorarbeiten zur Scheuchzerschen Korrespondenz liegen im Rahmen meiner Dissertation vor, die sich vorrangig mit der Sintfluttheorie Scheuchzers und den europäischen Debatten der Erdwissenschaften und Natur-

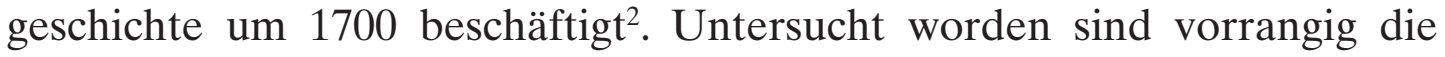
Verbindungen des Zürcher Mediziners zur englischen Royal Society, die vor allem dem Transfer von Naturalien und Informationen dienten. Für die gelehrte Gesellschaft in London wurde Scheuchzer zum wichtigsten Informanten wissenschaftlicher Nachrichten über die alpine Welt zu Beginn des 18. Jahrhunderts ${ }^{3}$.

Vor dem Hintergrund dieser Vorarbeiten soll im Folgenden ein erster Überblick der Scheuchzer-Korrespondenz hinsichtlich medizingeschichtlicher Aspekte gegeben werden. Die Aussagen sind sowohl quantitativ wie qualitativ noch vorläufig. Eine detaillierte Gesamtanalyse wäre erst auf der Basis eines gründlichen Repertoriums möglich, wie es für die Korrespondenz Albrecht von Hallers vorliegt ${ }^{4}$. Die Voraussetzungen für ein solches Projekt

1 Johann Jakob Scheuchzer an Theodor Zwinger, 10.2.1700, in: Portmann 1964, 15.

2 Kempe 2003.

3 Vgl. Kempe 2000.

4 Boschung et al. 2002. 
wären durch die von Rudolf Steiger erstellten Verzeichnisse des ScheuchzerNachlasses in der Zentralbibliothek Zürich durchaus gegeben ${ }^{5}$. Auf dieser Folie soll hier das Themengebiet umrissen werden, um erste Anknüpfungspunkte für weitere Forschungsarbeiten bereitzustellen. Zum einen geht es darum, die Materialbasis für vergleichende Briefnetzforschungen zu erweitern, zum anderen im systematischen Sinne um eine Präzisierung der Frage, was damit gemeint sein kann, wenn von «Medizin in Briefnetzen» die Rede ist.

Der Text gliedert sich in vier Teile. Nach einführenden biographischen Hintergrundinformationen zur Person Scheuchzers, vor allem in seiner Funktion als Mediziner, soll in den weiteren drei Abschnitten der Frage nach «Medizin in Briefnetzen» jeweils auf verschiedenen Ebenen nachgegangen werden, um unterschiedliche Aspekte des Themenfeldes perspektivieren zu können. Zunächst wird auf die Personen fokussiert und - mit Blick auf Scheuchzer - nach dem «Briefwechsel der Mediziner» gefragt. Dann soll auf die Inhalte eingegangen und «Medizinisches im Brief» untersucht werden. Abschliessend gilt es, die Ebene der Strukturen anzuvisieren, indem Funktion und Bedeutung der «Briefkommunikationen für die Wissenschaftskultur der Medizin» analysiert werden. Auf diese Weise soll der Gegenstand «Medizin in Briefnetzen» eine mehrdimensionale Gestalt annehmen.

\section{Johann Jakob Scheuchzer als Mediziner}

Scheuchzer wurde am 2. August 1672 als Sohn eines Stadtarztes in Zürich geboren ${ }^{6}$. Von 1692 bis 1695 studierte er Medizin, Botanik, Mathematik und Astronomie in Altdorf (bei Nürnberg) und im holländischen Utrecht. 1695 wurde er Waisenhausarzt und zweiter Stadtarzt in Zürich, zwei Jahre später Kurator der Bürgerbibliothek und Verwalter der städtischen Kunst- und Naturalienkammer. Im Collegium der Wohlgesinnten, einem vertraulichen Diskussionszirkel Zürcher Intellektueller, hielt er seit 1695 wissenschaftliche Vorträge ${ }^{7}$. Zwischen 1694 und 1711 unternahm er zwölf längere, zumeist mehrwöchige wissenschaftliche Alpenreisen. 1710 fand er an der Hohen Schule Zürichs, dem Collegium Carolinum, eine Anstellung als Mathematiklehrer. Dort sammelte er im Laufe der Jahre einen grossen Kreis von Schülern um sich. Zu den bedeutendsten zählen der durch die Zürcher Auf-

5 Steiger 1924; Steiger 1933.

6 Zur Biographie Scheuchzers mit weiterführenden Literaturhinweisen vgl. Kempe 2003, 22-28.

7 Kempe/Maissen 2002. 
klärungsliteratur bekannte Mediziner Laurenz Zellweger, ferner Johannes Gessner, der 1746 in Zürich die Physikalische Gesellschaft gründete, und Johann Jakob Leu, der Verfasser des Allgemeinen Helvetischen Eydgenössischen Lexikons. Auf diese Weise beeinflusste Scheuchzer direkt die Schweizer Aufklärung.

Scheuchzer war Mitglied der wichtigsten Akademien Europas ${ }^{8}$ und publizierte in den renommiertesten internationalen Wissenschaftszeitschriften ${ }^{9}$. Trotz seiner wissenschaftlichen Anerkennung im Ausland blieb ihm lange die angestrebte Physikprofessur am Collegium Carolinum, die höchste institutionelle Auszeichnung für einen Naturwissenschaftler in Zürich, versagt. Die Gründe hierfür waren immer wieder aufflammende Konflikte mit den staatlichen und kirchlichen Behörden. So stand er etwa in der Zürcher Unruhe von 1713 als Sprecher der bürgerlichen Oppositionspartei an der Spitze der aufständischen Zünfte. Erst 1729 wurde ihm erlaubt, am Carolinum auch naturwissenschaftliche Vorlesungen zu halten, und erst im Januar 1733 erhielt er den lebenslang ersehnten Posten als leitender Stadtarzt und Physikprofessor in Zürich. Fünf Monate später, am 23. Juni, starb er nach kurzer Krankheit.

Erscheinung und Persönlichkeit Scheuchzers (Abb. 1) beschrieb der Königsberger Physiker Christian Gabriel Fischer nach seinem Besuch in Zürich Ende August 1730 mit den Worten:

\footnotetext{
Seine Statur ist klein; seine Constitution gesund und seine Hand sehr flüchtig. Im Concipiren fehlt es ihm nicht an Einfällen, daher er im Schreiben reicher an Gedanken als in der Conversation ist. Seine Kleidung ist schlecht, doch rein. Die Conduite ehrlich, simple, sonder Ambition und vanité. Seine einzige Passion ist viel Wissen und schreiben, darauf hat er alle Zeit und sein Vermögen gewandt. Er leidet zwar keine Noth, hat aber auch nicht viel übrig, jedennoch ist er mit seinem Glück zufrieden. ${ }^{10}$
}

Im Mittelpunkt seines vielseitigen Schaffens stand die wissenschaftliche Erforschung der Schweizer Gebirgswelt. Mit Barometer, Winkelmessgradbogen, Gesteinshämmerchen, Thermometer und anderen physikalischen Instrumenten ausgerüstet, bereiste er die Alpen. Thematisiert wurden dabei alle sammelnden und ordnenden Teildisziplinen der Naturgeschichte: Botanik, Zoologie, Mineralogie und physische Geographie. 1712 fertigte er eine Karte der Schweiz an, die Nova Helvetiae tabula geographica. Sie wurde im 18. Jahrhundert mehrfach nachgestochen und blieb bis zu Beginn des 19. Jahr-

8 Academia Naturae Curiosorum (Leopoldina) in Schweinfurt (seit 1697); Royal Society in London (seit 1704); Preussische Akademie der Wissenschaften in Berlin (seit 1705); Accademia degli Inquieti in Bologna (seit 1706); korrespondierendes Mitglied der Académie des Sciences in Paris (seit 1707).

9 So etwa in den Londoner Philosophical Transactions, der Pariser Histoire de l'Académie Royale des Sciences oder den Berliner Miscellanea Berolinensia.

10 Fischer 1972, 2. 


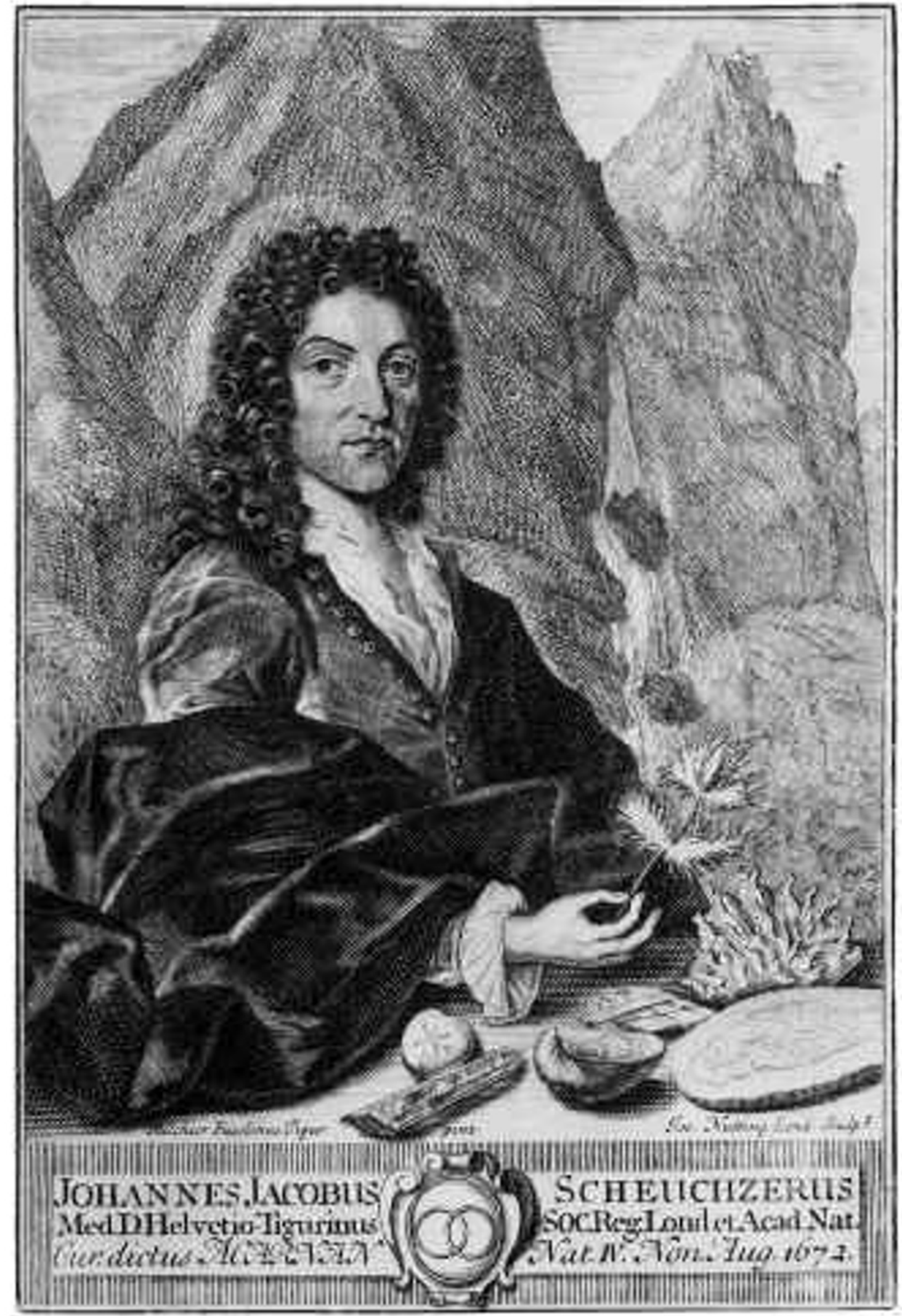

Abb. 1. Johann Jakob Scheuchzer im Alter von ca. 35 Jahren. Aus Scheuchzers Itinera alpina (London 1708) (Zentralbibliothek Zürich, Sig.: 7.33). 
hunderts die einschlägige Gesamtkarte der Schweiz. Darüber hinaus sammelte Scheuchzer auf seinen Alpenreisen auch als Historiker Materialien für seine geplante Historia Helvetiae und durchwanderte das Alpenland mit den Augen eines Ethnographen, der die Sitten und Bräuche der verschiedenen Alpenvölker studierte. Schliesslich plante Scheuchzer eine «Anthropologie der Alpenmenschen», eine Untersuchung über die körperlichen, mentalen und moralischen Eigenschaften der Schweizer Bergbewohner, die jedoch nie zur Ausführung gelangte. Scheuchzers populärwissenschaftliche Reiseberichte dienten sowohl Naturforschern als auch den ersten Alpentouristen als Reiseführer. Sie wurden zu einem wichtigen Multiplikator der ab Mitte des 18. Jahrhunderts in ganz Europa einsetzenden Alpenbegeisterung, insbesondere des Philhelvetismus der deutschen Aufklärung.

Wissenschaftsgeschichtlich von Bedeutung sind Scheuchzers Impulse für die alpine Hydrographie, Balneologie, Glaziologie, seine Vorarbeiten auf dem Gebiet der alpinen Botanik sowie seine barometrischen Höhenmessungen und meteorologischen Beobachtungen in den Hochgebirgsregionen. Vor allem aber spielte Scheuchzer eine Schlüsselrolle für die moderne Geologie und Paläontologie. Die in diesem Zusammenhang entworfene Sintfluttheorie entwickelte erstmals ein Stratifikationskonzept zur Untersuchung von Gesteinsschichten. Insbesondere aber verhalf sie der Erkenntnis zum endgültigen Durchbruch, dass Fossilien keine «Naturspiele», sondern versteinerte Überreste oder Spuren von Tieren und Pflanzen sind, die einst wirklich gelebt haben ${ }^{11}$.

Scheuchzers Tätigkeiten als theoretischer und praktischer Mediziner sind in der Forschung bislang nur wenig thematisiert worden ${ }^{12}$. Auf dem Feld der Theorie hat sich Scheuchzer mit allen grundlegenden Gebieten der Medizin beschäftigt, methodisch ging er dabei häufig kasuistisch vor, wie etwa 1732 in der Beschreibung des «Fliegenden Zungenkrebses», der Maul- und Klauenseuche. Zu Unrecht gilt der Zürcher Mediziner in der Forschung als reiner Iatromechaniker ${ }^{13}$. In der Pharmacia Contracta, einem handschriftlichen Arzneiverordnungsbuch, übernimmt Scheuchzer das Rezept «Elixir Proprietatis Paracelsis» ${ }^{14}$. Bei der Wiedergabe des Rezeptes dieser Arznei, die vorrangig die Drogen «Myrrha Alexandrina», «Aloes Epatica» und «Crocus Orientalisseu Ungaricus» enthält, verwendet Scheuchzer alchemistische Zeichen und Symbole und empfiehlt die Herstellung des Elixiers mit «Spiritus Sulphuris» oder «Sal comune Tartari». Scheuchzer erweist sich hier als

11 Vgl. ausführlich Kempe 2003.

12 Ein kaum in die Tiefe gehender Überblick zum Thema 〈Scheuchzer als Arzt〉 findet sich bei Fischer 1973, 112-119.

13 Beerli 1980.

14 Büchi 1982. 
Anhänger der in der paracelsischen Medizin des 16. und 17. Jahrhunderts eingeführten anorganischen Stoffe. Als Mediziner und Pharmazeut war Scheuchzer daher auch von der Iatrochemie beeinflusst.

Scheuchzer beschrieb Mutterkornvergiftungen, warnte wiederholt vor Quecksilbersalben, purgierenden Mitteln oder der übertriebenen Anwendung des Aderlasses. Auf seinen Schweizer Alpenreisen besuchte der Zürcher Mediziner Heilbrunnen, Thermen und Bäder, als Badearzt konnte er auf eigene therapeutische Erfahrungen zurückgreifen. Auf einer Alpenreise behandelte Scheuchzer die Mönche des Klosters Engelberg, welche an einer schweren Darmkolik litten. Zahlreiche Schüler wurden von ihm in Privatkollegien unterrichtet - anatomische Sektionen mit eingeschlossen - und so auf das Medizinstudium im Ausland vorbereitet. Im Toggenburger Krieg von 1712 diente Scheuchzer als Feldarzt der Zürcher Truppen.

In seiner amtlichen Tätigkeit war Scheuchzer auch für die Prophylaxe von Epidemien zuständig, als praktizierender Arzt behandelte er ebenso Fremde, die auf der Durchreise in Zürich erkrankt waren. So etwa 1705 den Engländer William Wyrdham, der Christian Kinnaird auf einem Grand Tour nach Italien begleitete und in Zürich erkrankte ${ }^{15}$. An einem spektakulären Beispiel lässt sich Scheuchzers Handeln als Amtsarzt besonders plastisch dokumentieren: 1701 fand im reformiert-orthodoxen Zürich der sogenannte «Wasterkinger-Handel», einer der letzten Hexenprozesse der Schweiz, statt: Die der Hexerei angeklagten elf Einwohner aus dem Dorf Wasterkingen wurden ins Gefängnis nach Zürich gebracht, wo eine Frau von ihnen unter der Folter Verkehr mit dem Teufel gestand. Das Resultat waren acht Todesurteile, sieben auf dem Schafott vollstreckt, eins auf dem Scheiterhaufen ${ }^{16}$. Noch während des laufenden Prozesses berichtete Scheuchzer im vertraulichen Gesprächskreis der Wohlgesinnten, dass er selbst vor kurzem zusammen mit dem Henker die im Gefängnis sitzenden Angeklagten aus Wasterkingen medizinisch untersucht habe. Die sonderbaren schwarz-blauen Flecken am Knie einer Frau hielt er jedoch nicht für teuflische Zauberzeichen, sondern bloss für eine gewöhnliche Fistelart, «ob schon der Henker ihro mit einer subtilen Nadel darein gestochen, ohne das sie etwas merklichs empfunden, weilen diß der orth ist aller dergleichen Narben, das sie geringen, oder gar keine schmerzen empfunden, ob man schon darnein hauet und sticht» ${ }^{17}$. Dass Scheuchzer die angeblichen Teufelszeichen lediglich als eine medizinisch beschreibbare Hautkrankheit einstufte, hatte den angeklagten Frauen aus Wasterkingen allerdings nicht geholfen.

15 Christian Kinnaird an Hans Sloane, 10.10.1705, in: BL Ms. Sl 4040, fol. 77r-78r, hier 77v-78r. 16 Vgl. Meili 1980.

17 Johann Jakob Scheuchzer, [Sitzungsprotokoll], 10.6.1701, in: ZBZ Ms. Z III 622, S. 155-159. 


\section{Der Briefwechsel der Mediziner}

Nähert man sich dem Thema «Medizin in europäischen Briefnetzen», so lässt sich auf einer ersten Ebene nach den Medizinern als Träger solcher Korrespondenzen fragen. Der Zürcher Waisenhausarzt stand in Korrespondenz mit zahlreichen Personen. In seinem umfangreichen Nachlass befinden sich 52 Bände mit etwa 5150 Briefen von ca. 700 Korrespondenten, Briefe von mindestens 100 weiteren Adressaten sind verlorengegangen. Daneben hinterliess Scheuchzer fünf Bände mit ca. 1800 Entwürfen oder Kopien der von ihm abgesandten Briefe ${ }^{18}$. Anzahl der Briefe und Korrespondenten liegen

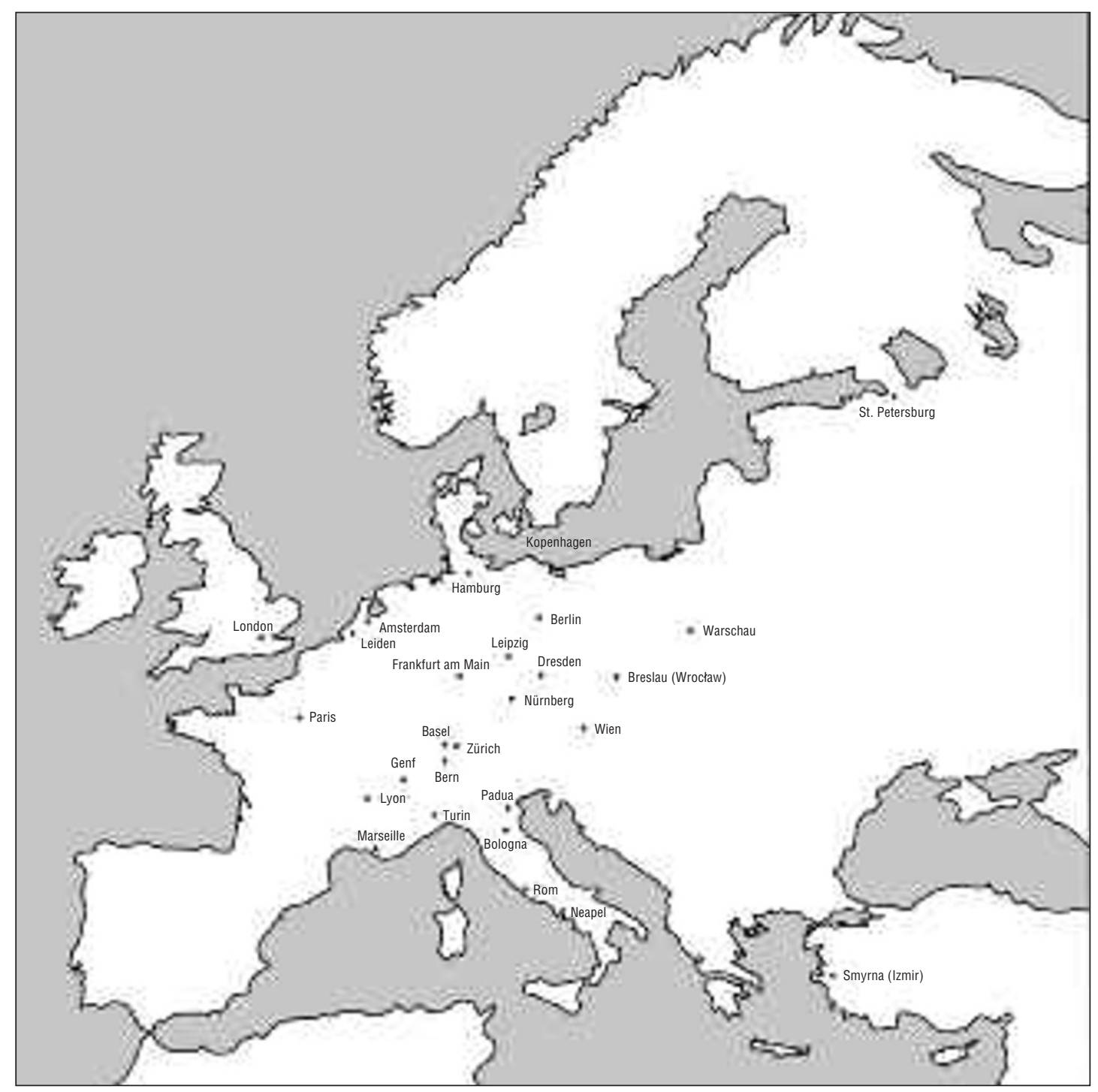

Abb. 2. Scheuchzers Korrespondenznetz, in Ausschnitten, geographische Ausbreitung.

18 Steiger 1933, 47. 

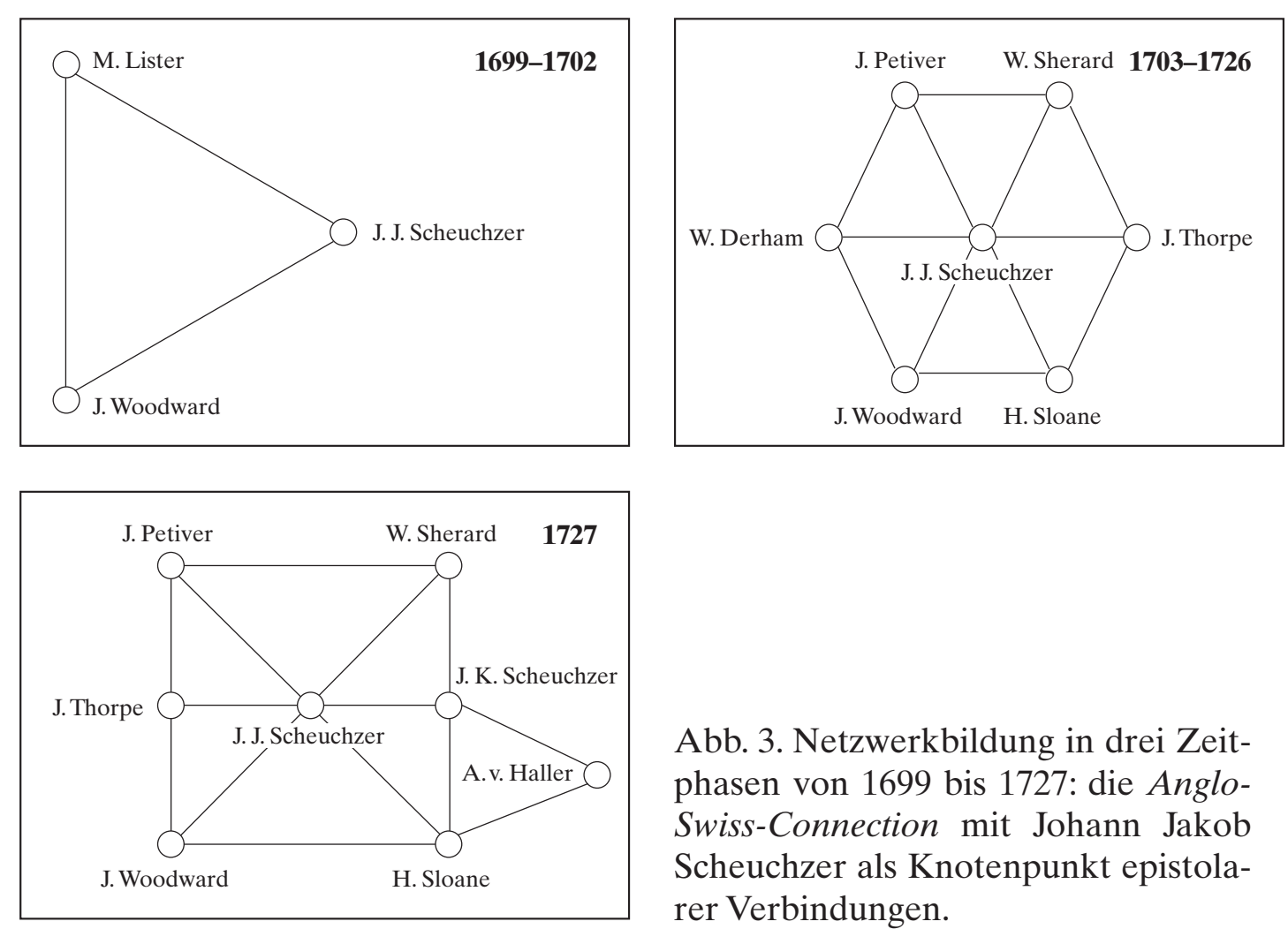

Abb. 3. Netzwerkbildung in drei Zeitphasen von 1699 bis 1727: die AngloSwiss-Connection mit Johann Jakob Scheuchzer als Knotenpunkt epistolarer Verbindungen.

etwas höher als zum Beispiel beim Nürnberger Stadtphysicus Christoph Jakob Trew (4800 Briefe an 700 Korrespondenten) ${ }^{19}$. Im Unterschied zu Trew kann man jedoch bei Scheuchzer wie bei Haller von einem Korrespondenznetz «europäischen Typs» sprechen. Geschätzte 60\% der Briefe stammen aus Ländern ausserhalb der Schweiz, vorrangig England, Deutschland, Italien, Frankreich, auch Österreich (Habsburg), Skandinavien und Russland (Abb. 2). Ein besonderer Korrespondenz-«Strang» bildete sich zwischen Scheuchzer und führenden Vertretern der Royal Society in London heraus, unter ihnen die Mediziner Martin Lister, John Woodward, Hans Sloane, William Sherard, John Thorpe und der Apotheker James Petiver. Scheuchzer wurde zu einem zentralen Verbindungsmann der Royal Society zur Schweiz. Indem dessen Sohn, Johann Kaspar Scheuchzer, dem jungen Haller den Kontakt zu Sloane, dem Präsidenten der Royal Society, vermittelte, sicherte er die Kontinuität der Anglo-Swiss-Connection seines Vaters über dessen Tod 1733 hinaus (Abb. 3) ${ }^{20}$.

Scheuchzer, Haller und der erwähnte Trew standen alle drei in brieflicher Verbindung. Scheuchzer und der erheblich jüngere Haller allein haben - nach

19 Zu Trew vgl. Schnalke 1997 und den Beitrag in diesem Heft von Marion Maria Ruisinger, Thomas Schnalke.

20 Vgl. Kempe 2000 und Kempe 2003, 73-109. 
Angaben der Datenbank des Berner Haller-Projektes - insgesamt 24 gemeinsame Korrespondenten. Diese Schnittmenge verdeutlicht die dichte Verzahnung der gelehrten Korrespondenz im 18. Jahrhundert. An den gemeinsamen Briefschreibern lässt sich auch der insgesamt hohe Anteil der Berufsgruppe der Mediziner ablesen. Von den 24 Scheuchzer-HallerKorrespondenten sind allein 15 Ärzte. Dieser Trend wird durch die Gesamtkorrespondenz Scheuchzers bestätigt. Zu den Berufsgruppen der Korrespondenten zählen - neben den Medizinern - auch Juristen, Theologen, Naturwissenschaftler, Historiker, Philologen und andere Akademiker, Gewerbetreibende (Verlagswesen, Buchhändler), Beamte, Politiker, Militär und Kaufleute. Die führende Berufsgruppe bilden jedoch mit 208 Korrespondenten die Mediziner. Prozentual entspricht dies etwa einem Anteil von $28 \%$ sämtlicher in der Scheuchzer-Korrespondenz vertretenen Berufsgruppen. Im einzelnen: 180 promovierte praktizierende Ärzte, Stadt-, Landund Leibärzte oder Medizinprofessoren, 11 Medizinstudenten, 9 Apotheker sowie 8 Chirurgen ${ }^{21}$.

Die Briefe zwischen Medizinern dienen - wie Thomas Schnalke für Christoph Jakob Trew gezeigt hat - als wichtige Quelle für das Selbstverständnis der Ärzte ${ }^{22}$. Entsprechendes kann auch für Scheuchzer nachgewiesen werden, vor allem im Briefwechsel zwischen 1700 und 1724 mit Theodor Zwinger III, der in Basel als Arzt praktizierte und dort Professor für Botanik und Anatomie war. Während Zwinger in seinen Briefen an Scheuchzer nicht müde wurde hervorzuheben, dass er sich in erster Linie der Medicina Praxi verpflichtet fühle, betonte letzterer immer wieder, er ziehe das Studium der Schweizer Naturgeschichte der praktischen Medizin eindeutig vor:

Den meisten Ärzten aber erscheint diese angenehme Nahrung meiner Seele [i.e. historia helvetiae naturalis, M. K.] töricht und ungewürzt; sie erachten sie für Brotgewinn weniger geeignet als die goldene Praxis, die sie zwar nicht besitzen, nach der jedoch diese Geizhälse mit offenem Rachen trachten. Ich jedoch widme dieser Tätigkeit und anderen derartigen Studien diejenigen Stunden, die mir andere notwendigere Dinge und meine Praxis (die zwar nur noch an einem dünnen Faden hängt) übriglassen; mein Schicksal zieht mich dazu $\operatorname{hin}[\ldots] \cdot{ }^{23}$

Als Scheuchzer 1712 von Gottfried Wilhelm Leibniz zum Leibarzt des Zaren Peter I. vorgeschlagen wurde, interessierten ihn nicht die Krankheiten der russischen Thronfamilie, sondern die naturgeschichtliche Erforschung der unbekannten Weiten Russlands. Nach der geplatzten Vokation schrieb

21 Die Zahlen dieser Übersichten basieren auf Angaben von Steiger 1933 und eigenen Berechnungen. Indes sind weitere Briefforschungen notwendig, um die Angaben zu spezifizieren und die Berechnungen fortzuführen.

22 Schnalke 1997.

23 Scheuchzer an Zwinger, 7.4.1700, in: Portmann 1964, 19, übersetzt aus dem Lateinischen $208 \mathrm{f}$. 
Scheuchzer im März 1713 an Leibniz: «Schon befand ich mich in Gedanken in Russland, sah mich von einer Stadt und Provinz in die andere fliegend, um vor allen anderen in der Terra Virginea neue Pflanzen zu beobachten, neue Mineralien und Tiere zu beschreiben [...].» ${ }^{24}$ Dementsprechend diente ihm das ausgedehnte internationale Korrespondenznetz vorrangig zum Transfer naturgeschichtlicher Informationen und Objekte (Pflanzen, Samen, Steine, Fossilien usw.). Von den oben aufgelisteten 180 Medizinern sind es vor allem diejenigen Ärzte, die sich neben ihrer medizinischen Tätigkeit auch und gerade der historia naturalis widmeten, mit denen Scheuchzer am intensivsten korrespondierte, das heisst über viele Jahre hinweg zahlreiche Briefe und Pakete austauschte. Unter ihnen zum Beispiel Johann Philipp Breyne, Karl Nikolaus Lang, Giovanni Battista Morgagni, William Sherard, Hans Sloane, Antonio Vallisneri und John Woodward.

Das verbindende Interesse für Naturgeschichte überstieg zudem die Berufsgruppengrenzen; es findet sich bei den Physikern genauso wie bei vielen Theologen, die mit Scheuchzer korrespondierten. Letztere bilden mit ca. 17\% (119 Pfarrer, Theologieprofessoren, Äbte, Mönche) nach den Medizinern die zweitgrösste Berufsgruppe der Scheuchzer-Korrespondenten.

Warum so viele Theologen? Die Antwort verweist auf einen wichtigen kommunikationsgeschichtlichen Umstand: Scheuchzer plante eine umfassende Naturgeschichte der Schweiz, und für seine projektierte historia helvetiae naturalis baute er ein eigenes schweizweites Informantennetz auf. Bevorzugte Informanten waren vor allem die Pfarrer und Geistlichen auf dem Lande, die Scheuchzer als Ansprechpartner für naturgeschichtliche Fragen dienten. Hierzu versandte er auch als Beilage einen gedruckten Einladungsbrief zur Erforschung natürlicher Wunderen, so sich im Schweizerland befinden mit 189 konkreten Fragen aus den unterschiedlichen Teilgebieten der Naturgeschichte, unter anderem Fragen nach dem Aussehen und Verhalten der Schweizer Drachen, den dracones helvetici ${ }^{25}$. Als Knoten- und Koordinationspunkte konnten weitere Korrespondenten zwischengeschaltet werden, so etwa der erwähnte Mediziner Zwinger, der seinerseits Rundschreiben an Pfarrer aus dem Basler Gebiet verschickte und die ihm zugesandten Mitteilungen über Bergnamen, Flussläufe, Naturkuriositäten und ähnliches dann an Scheuchzer weiterleitete ${ }^{26}$.

24 «Translatus jam mente videbar in Russiam, videbar mihi ab una urbe et Provincia volans in alteram, una in Terrae virgineae novas observare Plantas, Mineralia nova et Animalia describere [...].» Johann Jakob Scheuchzer an Gottfried Wilhelm Leibniz, 20.3.1714, NLH Ms. LBr. 809, fol. 91r.

25 Scheuchzer 1699.

26 Vgl. Portmann 1964, 214 und 217. 


\title{
Medizinisches im Brief
}

Neben den Personen lässt sich nun auch - auf einer zweiten Ebene der Analyse - nach den Inhalten fragen: Welche medizinischen Themen und Sachfragen kommen in Scheuchzers Korrespondenzen mit Medizinern zur Sprache? In den Briefen thematisiert werden zum Beispiel medizinische Theorien, etwa zur Deutung der Pest, über die Ursachen der Pocken, der Kropfbildung oder zu verschiedenen Formen hohen Fiebers. Diskutiert wird über das «Heimweh» als spezifische Krankheit der Schweizer, über Heilund Mineralwasser oder über den Nutzen der physikalisch-mechanischen Medizin. Sichtbar werden darüber hinaus medizinische Deutungsmuster und Weltbilder. So erstaunt es, dass Scheuchzer, der sich in seinen Drucken vehement gegen den traditionellen Prodigienglauben wendet ${ }^{27}$, zugleich in einem Brief an Zwinger angesichts einer aktuellen Pestgefahr miasmatheoretische Annahmen vertritt, die einer solchen Zeichentheologie nahekommen. Er glaube nicht an ein Nachlassen der Pest, denn:

\begin{abstract}
die Meteorologie des vergangenen und gegenwärtigen Jahres scheint mir nichts Gutes zu verkünden: im vergangenen Jahre eine fliegende Feuerkugel, mehrere feurige Meteore; ein sehr heißer Sommer; ein Heuschreckenschwarm, der jene selbe Provinz, die jetzt von der zerstörerischen Pest heimgesucht wird, überfiel; andernorts eine ungeheure Menge anderer Insekten; darauf folgend ein milder Winter; die Beschaffenheit des gegenwärtigen Jahres überaus feucht; in der ganzen Schweiz häufige und verschiedenartige Fieber; in Luzern überaus zahlreiche Semitertianfieber, in der Rheingegend verschiedene andere Fieber; überall bei uns zahlreiche Raben; diesen Zeichen sollen, wie zu lesen ist, verderbliche Seuchen vorangegangen sein. ${ }^{28}$
\end{abstract}

Grundsätzlich gilt, dass die «Medizin» in der Korrespondenz nur ein, zugleich nachgeordnetes Themenfeld bildet. Die in Scheuchzers Briefen vertretenen Wissensfelder lassen sich - im idealtypischen Durchschnitt betrachtet - wie folgt prozentual einteilen: ca. 50\% Naturgeschichte (Mineralogie, Botanik, Zoologie und physische Geographie); ca. 15\% Mathematik, Physik und Astronomie; ca. 10\% Medizin; ca. 10\% Geschichte und Politik; ca. 10\% Bibliographie, Museographie und Kartographie; sowie ca. 5\% «PhysikoTheologie».

Entscheidend ist, dass im Kommunikationsraum «Korrespondenz» verschiedene Wissensdisziplinen aufeinandertreffen. Beobachten lässt sich hier - zumindest bis zu einem gewissen Grad - das Zusammenspiel unterschiedlicher Disziplinen. Zum Beispiel zeigt der Austausch botanischer Informationen, der Transfer von Pflanzen und Samen mit eingeschlossen, dass die «Botanik» als Disziplin genau an der Schnittstelle zwischen Medizin und

27 Vgl. Kempe 2003, 262-274.

28 Scheuchzer an Zwinger, September 1720, Portmann 1964, 191, übersetzt aus dem Lateinischen 281 . 
Naturgeschichte steht. Erstere wird bedient, wenn beispielsweise Hermann Boerhaave in Leiden von Scheuchzer Samen alpiner Heilpflanzen für seinen hortus medicus erhält ${ }^{29}$, letztere dagegen, wenn Scheuchzer botanische Informationen sammelt, um seine Naturgeschichte der Schweizer Pflanzenwelt vorzubereiten. Erkundigt sich Scheuchzer brieflich nach dem Gesundheitszustand der Alpenbewohner unterschiedlicher Regionen, dann steht hier das Medizinische nicht für sich, sondern bloss im Dienst einer geplanten «Anthropologie» der Alpenvölker als Teilgebiet einer auch die Natur des Menschen umfassenden Naturgeschichte der Schweiz.

Zum «Medizinischen» gehören neben wissenschaftlichen Inhalten im weiteren Sinne auch Reflexionen über die soziale und gesellschaftliche Stellung der Mediziner sowie allgemeine Nachrichten über das Gesundheitswesen. Immer wieder spricht sich Scheuchzer entschieden gegen die «medicastri empirici» oder «Quacksalber» aus. Seinen Korrespondenten berichtet er über den von ihm in Zürich ausgelösten «Halskragenstreit», als er sich wiederholt weigerte, bei Krankenvisiten den Mühlsteinkragen zu $\operatorname{tragen}^{30}$. Theodor Zwinger III betont die Unabhängigkeit der Medizinprofessoren, die im Unterschied zu den Hofärzten, Archiatern und Poliatern nicht unter dem Befehl kirchlicher oder politischer Behörden zu stehen hätten $^{31}$. Von Zwinger erfährt Scheuchzer auch von den Plänen des französischen Hofarztes Chirac, eine Akademie der praktischen Ärzte zu gründen, um alle medizinischen Beobachtungen und Erfahrungen zentral bündeln und auswerten zu können ${ }^{32}$.

\section{Bedeutung und Funktion der postalischen Kommunikation für die Wissenschaftskultur der Medizin}

Nach den Personen, den Inhalten sollen nun - auf einer dritten Analyseebene - die Funktionen der Korrespondenzkommunikation näher in den Blick genommen werden. Für die Medizin lassen sich in der Scheuchzer-Korrespondenz folgende vier Funktionsbereiche festmachen:

29 Vgl. Lindeboom 1962-1979; Belloni 1974.

30 Scheuchzer an Zwinger, 8.2.1713, in: Portmann 1964, 157.

31 Zwinger an Scheuchzer, 6.10.1720, in: Portmann 1964, $192 \mathrm{f}$.

32 Zwinger an Scheuchzer, 25.7.1719, in: Portmann 1964, 188. 
Das sind die zentralen Funktionen des Postverkehrs. Dazu zählt nicht nur der Austausch wissenschaftlicher Informationen und gesundheitspolitischer Nachrichten, sondern ebenso auch der Austausch didaktischer Erfahrungen als Lehrer medizinischer Collegien oder die Vermittlung von Erfahrungen in der medizinischen Praxis, etwa über Anwendungen von Heilwasser, Praktiken anatomischer Sektionen oder Behandlungen hohen Fiebers. Über seine ersten Erfahrungen in der ärztlichen Praxis in Trogen berichtet Zellweger seinem Lehrer Scheuchzer, er habe ein hitziges Fieber «glücklich curiert, jedoch wollen die Leut daran nicht kommen, sondern in der Aderlässe dem alten Gebrauch nach, Wein trinken und sich lieber den ordonanzen eines alten Weibs oder medicastri empirici underwerffen, so dass ich dess practicieren schier müd worden bin $»^{33}$.

Zentral ist, dass neben den Mitteilungen im Brief ebenso Wissensvermittlungen in Form von Beilagen oder Paketen hinzukommen. Den Briefen beigelegt wurden häufig Handschriften und Drucke (etwa medizinische Dissertationen, die der Korrespondent als Präses verfasst hatte), aber auch Blumensamen oder getrocknete Pflanzen. Zur Publikation bestimmte Texte wurden oft befreundeten Korrespondenten zur Korrektur geschickt. Beigelegte Drucke sollten verschenkt, getauscht, verkauft oder auch nur verliehen werden. Viele heute erhaltene Briefe sind sogar ursprünglich nur Begleitschreiben grösserer Pakete gewesen. Solche in den Netzverbindungen kursierende Pakete konnten die verschiedensten Dinge enthalten: neben Druckwerken und Pflanzen auch Fossilien, Mineralien, Kristalle, Mikroskoppräparate, Münzen, Abbildungen, Karten, Porträts, Medaillen, archäologische Funde, medizinische Heilmittel, wissenschaftliche Instrumente oder handwerkliche Produkte. Über zwei Jahrzehnte hinweg verschoben Scheuchzer und der englische Mediziner und Naturforscher John Woodward zwischen London und Zürich regelmässig grössere Fossilienkisten ${ }^{34}$. Oft erscheinen die Briefe gegenüber den sie begleitenden Paketen in ihrer Bedeutung sogar als nachrangig.

Der Begriff «Korrespondenz» meint hier also eine Brief und Paket umfassende Form postalischer Kommunikation. Pakete und Beilagen erhöhten den Wissensgehalt enorm, Briefbeilagen ermöglichten zudem sowohl eine Weiterversendung an andere Personen durch sekundäre Zirkulationen und

33 Zellweger an Scheuchzer, 31.3.1714, in: Schudel-Benz 1924, 46.

34 Vgl. Kempe 2000 und Kempe 2003, 73-109. 
Distributionen als auch eine parallele Verschickung serieller Informationen - letzteres bei Scheuchzer etwa in Form von Tabellen seiner barometrischen Höhenmessungen. Im Rahmen der Korrespondenznetze konnten auf diese Weise synchrone Wissensströme entstehen und sich rasch ausbreiten. Es ist daran zu erinnern, dass sich der expandierende Buchmarkt noch zu Beginn des 18. Jahrhunderts zu grossen Teilen über den postalischen Verkehr organisierte. Für die Distribution wichtiger medizinischer Werke übernimmt Scheuchzer eine zentrale Steuerungsfunktion. So erhält er beispielsweise postalisch von Giovanni Battista Morgagni aus Venedig dessen Adversaria anatomica prima (Bologna 1706) in zwei Exemplaren, um eines davon an den Genfer Arzt Jean-Jacques Manget weiterzuleiten ${ }^{35}$.

\section{Frühwarnsystem für Epidemien}

Eine besondere Form des Wissenstransfers stellt die Frühwarnung dar. Als zweiter Stadtarzt war Scheuchzer auch für den Schutz Zürichs vor Epidemien und Seuchen verantwortlich. Mittels seiner Korrespondenzen erhält Scheuchzer immer wieder Hinweise anderer Ärzte aus der Schweiz, Deutschland, Frankreich oder Italien, ob eine Viehseuche ausgebrochen sei oder ob eine aufgetretene Fieberkrankheit ansteckend sei oder nicht. Nachdem 1720 in der Gegend von Marseille eine verheerende Pestwelle ausgebrochen war, nahm Scheuchzer brieflich Kontakt mit südfranzösischen Pestärzten auf. Die Briefwechsel dienten nicht nur der Beschaffung von Informationen über das Krankheitsbild, über Vorbeuge- und Behandlungsmassnahmen vor Ort, sie dienten zugleich auch der ständigen Überwachung der räumlichen Ausdehnung und Verbreitungsgeschwindigkeit einer solchen Epidemie. Dem zugute kam vor allem die Netzwerkstruktur der Korrespondenzen. So wurde Scheuchzer auch über andere Briefkanäle vom Verlauf der Pest in der Provence informiert. «Daß die Pest in den französischen Provinzen stark abgenommen hat, haben wir aus mehreren nach Basel überschickten Briefen mit großer Freude vernommen [...]», schreibt Zwinger am 7. Januar 1722 an seinen Kollegen in Zürich ${ }^{36}$.

35 Morgagni an Scheuchzer, 24.3.1707, und Scheuchzer an Morgagni, 1.6.1707, in: Belloni 1975, $28-30$.

36 Zwinger an Scheuchzer, 7.1.1722, in: Portmann 1964, 197, übersetzt aus dem Lateinischen 283. 


\section{Wissenschaftspolitik}

Der Informationstransfer beschränkt sich keineswegs auf die genannten Bereiche. Vielmehr fungieren die Briefwechsel auch als Instrument wissenschaftspolitischer Organisationen. Dabei geht es beispielsweise um die Abstimmung von Interessen bei Lehrstuhlbewerbungen für Medizinprofessuren, um das Einholen von Unterstützung solcher Bewerbungen oder um das Ausloten von Publikationsmöglichkeiten. Eine ebenso wichtige Rolle spielen die Briefe als Vermittlungsorgan für den Austausch von Schülern. Zwinger schickt einen seiner Söhne zur Vorbereitung seines Medizinstudiums zu Scheuchzer, der ihn in sein Haus aufnimmt und in Privatcollegien unterrichtet. Zuvor waren bereits einige Medizinschüler Scheuchzers auf dessen Empfehlung zu Zwinger nach Basel gekommen. Überbringt ein Schüler ein solches Empfehlungsschreiben selbst, wird der Brief zum Mittel der persönlichen Kontaktaufnahme, der Bote damit gleichsam Teil der überbrachten Kommunikation ${ }^{37}$.

\section{Fernkonsultationen}

«Consultes» räumlicher Distanzen wären noch näher zu untersuchen. Zumindest einen Hinweis auf eine erwünschte Fernbehandlung konnte ich nachweisen. Von Leibschmerzen geplagt, bittet ein Arzt aus Tirol den jungen Mediziner Laurenz Zellweger um den zusätzlichen Rat eines erfahrenen Arztes. So wendet sich Zellweger am 9. Juni 1715 brieflich an seinen Lehrer Scheuchzer:

Vorige Wochen wurde ich zu einem tyrolischen med. Doct. berufen, welcher vor incirca 8 Jahren, wegen vielen zu Pferd getanen Reyssen ein Herni am scrotalem bekommen hat, vor 4 Jahren hat ein Argwohn ihn eingenommen, als wann alle Menschen schier seine Feinde wären, hat dessentwegen ihr commercium geflohen und sich in seinem Haus aufgehalten, allzeit förchtende, dass wann er unter die Leut käme, ihn jemand fange, oder gar um das Leben bringen möchte. Vor weniger Zeit nun, haben ihm die Wind und andere Sachen erschröckliche Tormina verursacht. Diesen Torminibus folgen horrores et frigus per totum corpus. Begehrt desswegen eines alten und erfahrenen medici Rath. Uebrigens ist dieser Patient von 45 Jahren fett, sehr blutreich, ein hypochondricus und guter Speisen wie auch starken Weins ziemlich gewohnt, er respiriert auch wohl und ist zum Zorn zu Zeiten auch ziemlich geneigt. Ich habe von sectionem vor allem und auch andere Sachen rathen wollen, er verlangt aber eines alten practici Rath, wollen desswegen meinen Hrn. Dr. gebeten haben, ihre Meinung darüber zu eröffnen. ${ }^{38}$

37 Zwinger an Scheuchzer, 28.4.1705, in: Portmann 1964, 120, übersetzt aus dem Lateinischen 253: «Endlich findet sich mein Sohn [Johann Jacob Zwinger, 1685-1708] mit diesem meinem Brieflein bei Ihnen ein; er fleht Sie bescheiden an, sein Gönner zu werden und ihm bei seinen Studien beizustehen. Auch ich bitte Sie sehr, ihm Ihren Unterricht zu erteilen.»

38 Laurenz Zellweger an Johann Jakob Scheuchzer, 9.6.1715, Schudel-Benz 1924, 50f. 
Die Multifunktionalität postalischer Kommunikationen spiegelt sich bereits in der - idealtypischen - Struktur gelehrter Briefe wider: ca. 25\% Wissenschaftsorganisation (Lehrstuhlbewerbungen, Studentenaustausch etc.); ca. $25 \%$ vermischte Nachrichten (lokale Nachrichten, gesellschaftliche oder politische Skandale etc.); ca. 25\% wissenschaftliche Inhalte; ca. 10\% Persönliches (Familie, Gesundheit); ca. 10\% Posttechnisches (Briefrouten, Paketinhalte, Bücherwunschlisten etc.); und ca. 5\% Höflichkeitsrhetorik. Die genannten Funktionen - Wissenstransfer und Kompetenzkommunikation, Frühwarnsystem für Epidemien, Wissenschaftspolitik und Fernkonsultationen - verweisen auf spezifische mediale Leistungen postalischer Kommunikationen: Das ist zum einen die epistolare Raum-Zeit-Überbrückung, die Frühwarnungen und Fernbehandlungen ermöglicht. Korrespondenzen verlängern wie ein Fernrohr den Radius der Wahrnehmung bei gleichzeitiger Einschränkung der haptischen Nähe, was im Fall einer Epidemie vor Ansteckung schützt. Des weiteren stellt der gelehrte Brief die Option eines multiplen Informationsvehikels bereit, um unterschiedliche Wissensträger (Briefmitteilungen, Beilagen, Objekte jeder Art) miteinander kombinieren und übertragen zu können. Ferner ist die Verzahnbarkeit mit anderen regionalen und überlokalen Medien, die mediale Scharnierfunktion des Briefes, hervorzuheben. Auf die enge Verknüpfung gelehrter Korrespondenzen mit wissenschaftlichen Zeitschriften ist schon mehrfach hingewiesen worden, auch auf die Verlängerung von Forschungsreisen in die Studierkammern der Daheimgebliebenen ${ }^{39}$. Ebenso lassen sich in Briefen lokale mündliche Kommunikationen bündeln und übertragen. Bei der Überbringung durch Boten werden zudem die beiden überlokalen Medien «Brief» und «Reise» miteinander verbunden.

Die mediale Leistung als Bindeglied unterschiedlicher Kommunikationssituationen verweist auf die eigentümliche Stellung des Briefes zwischen Schriftlichkeit und Oralität. Auf der einen Seite bleibt durch die Bindung an einen oder mehrere Absender die dialogische Struktur des Gespräches erhalten, auf der anderen Seite wird die Flüchtigkeit mündlicher Unterredung durch ihre Verschriftlichung aufgehoben. Der briefliche Verkehr nimmt damit eine Zwitterstellung zwischen Privatheit und Öffentlichkeit ein. Im Unterschied zur privaten Situation des Gesprächs gewinnt das im Brief Ausgedrückte allein durch die schriftliche Fixierung einen objektiven und prinzipiell öffentlichen Charakter, im Unterschied aber zum Aufsatz oder Buch bleibt durch die Verbindung von Absender und Adressat in gewisser Hinsicht das subjektive Moment einer privaten Gesprächssituation erhal-

39 Vgl. Stuber 2000. 
$\operatorname{ten}^{40}$. Zugleich wird umgekehrt auch bereits gespeichertes oder verfestigtes Wissen wieder «verflüssigt», wenn etwa durch das Verschicken von Objekten Naturaliensammlungen aufgelöst oder beigelegte Texte durch ihren Transfer der Kommunikation zugänglich und durch ihre Kommentierung dialogisiert werden. Aufgrund der Vernetzung der Korrespondenzen werden Informationen gebündelt und neu aufgefächert. Die Knotenpunkte, die Korrespondenten, fungieren als Multiplikatoren und erzeugen durch Adressatenbindungen zielgenaue, kontrollierte und selektive Streuungseffekte. Die Netzstruktur gewährleistet zudem, dass bestehende Hindernisse umgangen werden können. Während des englisch-französischen Krieges fungierte Scheuchzer in Zürich als Korrespondenzdrehscheibe zwischen der Royal Society in London und der Académie des sciences in Paris ${ }^{41}$.

Aber auch auf die «medialen Schattenseiten» postalischer Vernetzung, auf ihre Grenzen und ihre kommunikativen Defizite sei hier hingewiesen. Medizinischen Fernkonsultationen zum Beispiel mangelt es an den beschränkten Möglichkeiten der Ferndiagnose und den mannigfachen Schwierigkeiten, die sich aus der Phasenverzögerung ergeben ${ }^{42}$. Gerade durch die mediale Elastizität, Dialogsituationen räumlich wie zeitlich zu «zerdehnen» ${ }^{43}$, werden eine Reihe von Problemen erzeugt. Dabei kommt zum Tragen, dass Korrespondenzen zwar Räumlichkeiten «horizontal» zusammenschliessen, dabei aber zugleich «vertikal» erhebliche Zeiträume aufgefaltet werden, in denen nicht geringe Dissonanzen entstehen können. So kommt es im Briefwechsel zwischen Woodward und Scheuchzer immer wieder zu Irritationen, weil Pakete und die sie ankündigenden Briefe, die auf unterschiedlichen Wegen verschickt werden, sich verspäten, verlorengehen, wieder auftauchen und sich dabei so überschneiden, dass phasenweise grosse Verwirrungen auftreten ${ }^{44}$.Zwinger beschwert sich darüber, die Einbände per Post erhaltener Bücher seien so sehr von Mäusen angenagt, dass er nicht daran zweifle, sie seien lange Zeit in irgendeinem Winkel des Transportwagens liegengeblieben ${ }^{45}$. Ein Fuhrmann weigert sich, ein Paket Scheuchzers

40 So formulierte bereits Georg Simmel 1992, 430: «Die Form der brieflichen Äußerung bedeutet eine Objektivierung ihres Inhaltes, die hier eine besondere Synthese einerseits mit dem Bestimmtsein für ein einzelnes Individuum bildet, andrerseits mit dem Korrelat dieses: der Persönlichkeit und Subjektivität, mit der sich der Briefschreiber, im Unterschiede vom Schriftsteller, gibt. Und gerade in der letzteren Hinsicht ist der Brief als Verkehrsform etwas ganz Einzigartiges.»

41 Vgl. Kempe 2000 und Kempe 2003, 73-109.

42 Vgl. auch den Beitrag von Séverine Pilloud, Stefan Hächler und Vincent Barras im vorliegenden Heft.

43 Im Sinne des Textbegriffes von Konrad Ehlich als «wiederaufgenommener Mitteilung» im Rahmen einer «zerdehnten Situation»; vgl. Ehlich 1983.

44 Vgl. Kempe 2000 und Kempe 2003, 73-109.

45 Zwinger an Scheuchzer, 14.12.1702, in: Portmann 1964, 83. 
zu versenden, da es zu klein sei und er für den Transport kleiner zerbrechlicher Dinge keine Verantwortung übernehmen könne ${ }^{46}$. Woodward beklagt sich bei Scheuchzer: «Your Letter had taken Wett, so that I could not read what you write about the Plague in France.» ${ }^{47}$ Missverständnisse können nicht sofort ausgeräumt werden. Laurenz Zellweger schreibt an Scheuchzer, er habe in seinem letzten Brief nicht von einer Lotterie gesprochen, sondern von einer cotterie, womit er die neugegründete literarische Gesellschaft der Mahlern in Zürich meint ${ }^{48}$. Eine schwer lesbare Schrift und die knappe Darstellung mancher Sachverhalte werden nicht selten mit dem eiligen Termin der Postkutschenabfahrt begründet. Über das im Medium der Post Kommunizierte verliert der Absender jegliche Kontrolle, so dass Kommunikation sogar ins Leere schiessen kann. Als Woodward von Scheuchzer eine Ladung eigener deutschsprachiger Werke erhält, schickt dieser sie - sichtlich überfordert - weiter an Hans Sloane und notiert auf einem beigelegten Zettel: «I do not understand one Word of German. If it will be of any Use to you, I shall be very well pleased.» ${ }^{49}$

\section{Fazit}

Die Untersuchung hat gezeigt, dass der medizinische Briefwechsel auf allen drei Ebenen - Personen, Inhalte und Funktionen betreffend - jeweils als ein spezifischer Teilbereich gelehrter Korrespondenz anzusehen ist. Wenn Scheuchzer in einem Brief an Hermann Boerhaave von der «Respublica Medicae» spricht ${ }^{50}$, ist diese zugleich auch als Teilrepublik der res publica litteraria zu verstehen, die im weitgespannten Netzwerk europäischer (teils auch amerikanischer) Korrespondenten eine imaginierte Lokalität für eine grenzüberschreitende Gelehrtengemeinschaft geschaffen zu haben beansprucht. Netzwerktheoretisch betrachtet, fungierte Scheuchzer als Informationsdrehscheibe, als ein Kommunikationsmultiplikator. Aus der Perspektive des epistolaren Kommunikationssystems gesehen, verliert der einzelne Gelehrte, der einzelne Mediziner an Bedeutung. Stirbt ein Mitglied der Gelehrtenrepublik, dann versucht man, via Korrespondenz den Erhalt oder die Aufteilung seiner Naturaliensammlung, Bibliothek und seines Nachlasses zu organisieren. Als 1709 Boerhaave den verstorbenen Pierre Hotton als

46 Scheuchzer an Zwinger, 9.12.1703, in: Portmann 1964, 95.

47 Woodward an Scheuchzer, 27.5.1721, in: ZBZ Ms. H 293, 37-42, hier 41.

48 Zellweger an Scheuchzer, 16.10.1721, in: Schudel-Benz 1924, 65.

49 John Woodward an Sir Hans Sloane, BL Ms. Sl 4044, 23.6.1716, fol. 193r-193v, hier fol. 193r.

50 Scheuchzer an Boerhaave, 2.3.1709, in: Belloni 1974, 85. 
Medizin- und Botanikprofessor an der Universität Leiden ersetzt, setzt Scheuchzer mit diesem den zuvor mit Hotton betriebenen Austausch von Büchern und Pflanzen nahtlos weiter fort. Die sich überlappenden Korrespondenzverbindungen sorgen dafür, dass über den Tod des einzelnen Gelehrten hinaus das Briefnetz als transpersonaler Raum der Kommunikation erhalten bleibt.

\author{
Abkürzungen \\ $\mathrm{BL}=$ British Library \\ $\mathrm{NLH}=$ Niedersächsische Landesbibliothek Hannover \\ ZBZ = Zentralbibliothek Zürich
}

\title{
Bibliographie
}

Beerli, Urs-Peter, Medizinisches in Scheuchzers Physica Sacra oder Kupferbibel (Zürich 1980) Belloni, Luigi (Hrsg.), «Aus dem Briefwechsel zwischen Herman Boerhaave und Johann Jakob Scheuchzer», in: Arie Gerrit Lindeboom (Hrsg.), Circam Tilliam. Studia Historiae Medicinae (Leiden 1974) 83-106

- (Hrsg.), «Aus dem Briefwechsel von G. B. Morgagni mit den Brüdern Johann Jakob und Johannes Scheuchzer», Acta Historica Leopoldina 9 (1975) 27-49

Boschung, Urs et al. (Hrsg.), Repertorium zu Albrecht von Hallers Korrespondenz 1724-1777, 2 Bde. (Basel 2002)

Büchi, Jakob, «Die wiederaufgefundene 〈Pharmacia Contracta〉 des Johann Jakob Scheuchzer (1672-1733)», Gesnerus 39 (1982) 145-169

Ehlich, Konrad, «Text und sprachliches Handeln. Die Entstehung von Texten aus dem Bedürfnis nach Überlieferung», in: Aleida Assmann/Jan Assmann/Christian Hardmeier (Hrsg.), Schrift und Gedächtnis (München 1983) 24-43

Fischer, Christian Gabriel, Reisetagebücher, Ausschnitte zit. in: [Anonymus], «Eine Schweizerreise mit Johann Jakob Scheuchzer», Der Zürcher Landbote 30 (28.7.1972) 2

Fischer, Hans, Johann Jakob Scheuchzer (2. August 1672-23. Juni 1733). Naturforscher und Arzt (Zürich 1973) (Neujahrsblatt der Naturforschenden Gesellschaft in Zürich)

Kempe, Michael, «Die <Anglo-Swiss-Connection〉. Zur Kommunikationskultur der Gelehrtenrepublik in der Frühaufklärung», in: Robert Seidel (Hrsg.), Wissen und Wissensvermittlung im 18. Jahrhundert. Beiträge zur Sozialgeschichte der Naturwissenschaften zur Zeit der Aufklärung (Heidelberg 2000) 83-104 (Cardanus. Wissenschaftshistorisches Jahrbuch der Universität Heidelberg, 1)

- Wissenschaft, Theologie, Aufklärung. Johann Jakob Scheuchzer (1672-1733) und die Sintfluttheorie (Epfendorf 2003) (Frühneuzeit-Forschungen 10)

- /Thomas Maissen, Die Collegia der Insulaner, Vertraulichen und Wohlgesinnten in Zürich 1679-1709. Die ersten deutschsprachigen Aufklärungsgesellschaften zwischen Naturwissenschaften, Bibelkritik, Geschichte und Politik (Zürich 2002)

Lindeboom, Gerrit Arie (Hrsg.), Boerhaave's Correspondence, 3 vols (Leiden 1962-1979)

Meili, David, Hexen in Wasterkingen. Magie und Lebensformen in einem Dorf des frühen 18. Jahrhunderts (Basel 1980)

Portmann, Marie-Louise v. (Hrsg.), Die Korrespondenz von Th. Zwinger III. mit J. J. Scheuchzer 1700-1724 (Basel/Stuttgart 1964)

Scheuchzer, Johann Jakob, Einladungs-Brief/zu Erforschung natürlicher Wunderen/so sich im Schweitzer-Land befinden (Zürich 1699) [Wiederabgedruckt in: Hansjörg Küster/Ulf Küster (Hrsg.), Garten und Wildnis. Landschaft im 18. Jahrhundert (München 1997) 14-31] 
Schnalke, Thomas, Medizin im Brief. Der städtische Arzt des 18. Jahrhunderts im Spiegel seiner Korrespondenz (Stuttgart 1997)

Schudel-Benz, Rosa (Hrsg.), «Reise des Laurenz Zellweger mit Dr. J. J. Scheuchzer; Zellwegers Briefe an Scheuchzer aus Leiden, 1710-12; Briefe Zellwegers aus Trogen an Dr. Scheuchzer», Appenzellische Jahrbücher 51 (1924) 1-75

Simmel, Georg, Soziologie. Untersuchungen über die Formen der Vergesellschaftung (Frankfurt a.M. 1992) (Gesamtausgabe, Bd. 11)

Steiger, Rudolf, Verzeichnisse zur Scheuchzer-Korrespondenz (Zürich 1924); ZBZ Ms. H 348a

- Verzeichnis des wissenschaftlichen Nachlasses von Johann Jakob Scheuchzer (1672-1733), Beiblatt zur Vierteljahresschrift der Naturforschenden Gesellschaft in Zürich 78, Nr. 21 (Zürich 1933)

Stuber, Martin, «Forschungsreisen im Studierzimmer. Zur Rezeption der Grossen Nordischen Expedition (1733-1743) bei Albrecht von Haller und Samuel Engel», Gesnerus 57 (2000) 168-181 